\title{
Exercise for the Treatment of Depression
}

\author{
J. Knapen ${ }^{1,2, *}$, D. Vancampfort ${ }^{1,2}$, B. Schoubs ${ }^{3}$, M. Probst ${ }^{1,2}$, P. Sienaert ${ }^{1}$, P. Haake ${ }^{1}$, J. Peuskens ${ }^{1,4}$ \\ and G. Pieters ${ }^{1,4}$ \\ ${ }^{1}$ University Psychiatric Centre K.U.Leuven, Campus Sint-Jozef Kortenberg, Leuvensesteenweg, 517, 3070 Kortenberg, \\ Belgium \\ ${ }^{2}$ Faculty of Kinesiology and Rehabilitation Sciences, Katholieke Universiteit Leuven, Tervuursevest, 101, 3001 Leuven, \\ Belgium \\ ${ }^{3}$ Psychiatric Hospital Sancta - Maria, Melveren centrum 111, 3800 Sint- Truiden, Belgium \\ ${ }^{4}$ Faculty of Medicine, Katholieke Universiteit Leuven, Herestraat 49, 3000 Leuven, Belgium
}

\begin{abstract}
Depression is a common mental disorder that presents with depressed mood, loss of interest or pleasure, feelings of guilt or low self-worth, disturbed sleep and/or appetite, low energy, and poor concentration. Depression is a widespread and often chronic condition. Lifetime prevalence estimates for major depressive disorder are approximately $15 \%$ to $20 \%$; 1-year prevalence estimates are $5 \%$ to $10 \%$. Moreover, depression is characterized by high rates of relapse: $22 \%$ to $50 \%$ of patients suffer recurrent episodes within 6 months after recovery.

Depression is associated with a high incidence of co-morbid somatic illness. Individuals suffering from major depression run a higher relative risk of coronary heart disease, type 2 diabetes and osteoporosis compared with the general population. In general, depressed individuals exhibit a less active life-style and have a reduced cardio-respiratory fitness in comparison with the general population. Strong evidence demonstrates that lack of physical activity is associated with an unhealthier body mass and composition, and a biomarker risk profile for cardiovascular disease, type 2 diabetes, and osteoporosis. A growing body of evidence suggests that exercise is an effective treatment for depression. For mild to moderate depression the effect of exercise may be comparable to antidepressant medication and psychotherapy; for severe depression exercise seems to be a valuable complementary therapy to the traditional treatments. Exercise training not only improves depression, but also produces 'positive side effects' on depression associated physical diseases and cognitive decline.
\end{abstract}

Keywords: Exercise, depression, cardiovascular disease.

\section{INTRODUCTION}

There have been over 25 years of systematic investigation examining the relationship between exercise, especially aerobic forms and weight training, and depression. Recent meta-analyses support the growing evidence that exercise may be an effective complementary therapy in treatment of depression. Depression is associated with a high incidence of co-morbid somatic illnesses. Individuals suffering from major depression run a higher relative risk of coronary heart disease (CHD), type 2 diabetes and osteoporosis compared with the general population. Depression also is associated with poor cognitive functioning. The aim of this review is to present a comprehensive overview of beneficial effects of exercise on mental (i.e. mood and cognitive functioning) as well as somatic health of depressed individuals. To the authors knowledge, this is the first review that discuses both mental and somatic health benefits of regularly exercise on depression.

*Address correspondence to this author at the University Psychiatric Centre K.U.Leuven, Campus Sint-Jozef Kortenberg, Leuvensesteenweg, 517, 3070 Kortenberg, Belgium; E-mail: jan.knapen@ faber.kuleuven.be
We identified the meta-analyses in the area of exercise/physical activity and depression from 1998 until 2008. Relevant studies were identified by searching PubMed, Medline, Sport Discus, and PsycInfo. Terms used in the search were 'depression', 'clinical depression', 'depressive symptoms', 'exercise', 'physical activity', 'meta-analysis'. We also identified the meta-analyses and single-studies on the theme depression as an independent risk factor for development of coronary heart disease, type 2 diabetes and osteoporosis. All studies that investigated the role of exercise in the association among depression and these diseases were included. Finally, literature was also identified by citation tracking using reference lists from selected papers.

\section{DIAGNOSIS OF MAJOR DEPRESSIVE DISORDER}

Depression is a common mental disorder that presents with depressed mood, loss of interest or pleasure, feelings of guilt or low self-worth, disturbed sleep or appetite, low energy, and poor concentration. The diagnostic criteria for major depressive disorder following the American Psychiatric Association [1] are presented in Box I. 
Box I. The American Psychiatric Association Criteria for Diagnosis of Major Depressive Disorder

Five (or more) of the following symptoms have been present during the same 2 -week period and represent a change from previous functioning; at least one of the symptoms is either (1) depressed mood or (2) loss of interest or pleasure.

1. Depressed mood, nearly every day during most of the day

2. Marked diminished interest or pleasure in almost all activities

3. Significant weight loss (when not dieting), weight gain, or a change in appetite

4. Insomnia or hypersomnia (excess sleep)

5. Psychomotor agitation or psychomotor retardation

6. Fatigue or loss of energy

7. Feelings of worthlessness or inappropriate guilt

8. Impaired ability to concentrate or indecisiveness

9. Recurrent thoughts of death, recurrent suicidal ideation

\section{EPIDEMIOLOGY OF DEPRESSION}

Depression is a widespread and often chronic condition. Lifetime prevalence estimates for major depressive disorder are approximately $15 \%$ to $20 \%$; 1-year prevalence estimates are $5 \%$ to $10 \%$. Moreover, depression is characterized by high rates of relapse: $22 \%$ to $50 \%$ of patients suffer recurrent episodes within 6 months after recovery. Women are twice as likely as men to be diagnosed with depression, with lifetime prevalence rates of $10 \%$ to $25 \%$ in women versus $5 \%$ to $12 \%$ in men. Although rates of depression do not appear to increase with age, depression often goes undertreated in older adults [2].

In 2000, depression was the leading cause of disability as measured by Years Lived with Disability and the 4th leading contributor to the global burden of disease [3]. The World Health Organization expects that depression by the year 2020 reaches the second place in the ranking of Disability Adjusted Life Years calculated for all ages, including both sexes [3].

\section{DEPRESSION AND PHYSICAL HEALTH}

Depression is associated with a high incidence of comorbid somatic illnesses. Individuals suffering from major depression run a higher relative risk of CHD, type 2 diabetes and osteoporosis compared with the general population.

\section{Depression as a Primary Risk Factor for Coronary Heart Disease}

Depression is an independent risk factor for the development of CHD [4]. A meta-analysis of 11 prospective cohort studies of initially healthy individuals indicated that depression conferred a relative risk of 2.69 for adverse cardiac events, including myocardial infarction and cardiac death [5]. The Framingham heart study analyzed the risk ratios (Odds Ratio: OR) for traditional risk factors as well as clinical depression in men. The relative risk of depression
$(\mathrm{OR}=2.69)$ was higher than that of traditional risks factors for CHD, such as hypertension $(\mathrm{OR}=1.92)$, elevated blood lipid levels $(\mathrm{OR}=1.74)$, smoking $(\mathrm{OR}=1.71)$, diabetes $(\mathrm{OR}$ $=1.47)$ and age $(\mathrm{OR}=1.05)[6]$.

\section{Depression as a Secondary Risk Factor for Coronary Heart Disease}

Depression is an even stronger risk factor for cardiac events in patients with established CHD. Prospective studies have shown that depression increases the risk for death or nonfatal cardiac events approximately 2.5 -fold in patients with CHD. For instance, Frasure-Smith, Lesperance, Talajic followed 896 patients with a recent myocardial infarction and found that the presence of depressive symptoms was a significant predictor of cardiac mortality after controlling for multivariate predictors of mortality $(\mathrm{OR}=3.29$ for women and 3.05 for men) [7].

Many factors can contribute to an increased risk for CHD in depressed patients. The concept of a bio-behavioural model to explain the relationship between depression and CHD is gaining support in the literature $[2,4,8]$. This model includes variables varying from an increased presence of classical risk factors for CHD (such as smoking, hypertension, diabetes and physical inactivity) to changes in the immune system, dysregulation of the autonomic nervous system and increased platelet reactivity and coagulation.

Depression is associated with unhealthy behaviours that are in turn associated with CHD risk, such as reduced treatment adherence, smoking, poor dietary patterns, heavy alcohol use, and physical inactivity [2,4,9]. Two surveys investigated the causal role of physical inactivity in the relationship among depression and CHD. Kamphuis, Geerlings, Tijhuis, et al. determined the prospective independent and combined effects of depressive symptoms and physical inactivity on the 10-year cardiovascular mortality in a cohort of elderly men $(n=909)$ [10]. The results showed that depressive symptoms in elderly men are associated with a lower level of physical activity, and that depressive symptoms $(\mathrm{OR}=1.42$ per increase of one standard deviation) and physical inactivity $(\mathrm{OR}=1.09$ per 30-minute decrease) both increase the risk of cardiovascular mortality. The highest risk for cardiovascular mortality was attributable to the combined effect of depressive symptoms with physical inactivity $(\mathrm{OR}=1.47)$. Men with more depressive symptoms and a low level of physical activity had a strongly increased risk of cardiovascular mortality $(\mathrm{OR}=4.22)$ in comparison with men with few depressive symptoms and a high level of physical activity. Whooley, de Jonge, Vittinghoff, et al. conducted a study to determine why depressive symptoms are associated with an increased risk of cardiovascular events in patients with stable coronary heart disease [11]. The study included 1.017 outpatients with average follow-up of 4.8 years. The researchers found that participants with depressive symptoms had a $50 \%$ greater risk of cardiovascular events: the ageadjusted annual rate of cardiovascular events was $10 \%$ among the 199 participants with depressive symptoms and $6.7 \%$ among the 818 participants without depressive symptoms. After further adjustment for health behaviours, including physical inactivity, there was no longer a significant association between depressive symptoms and cardiovascular events. Participants with depressive symptoms had a $50 \%$ 
greater inactivity than participants without depressive symptoms. In the depressed group, physical inactivity was associated with a $44 \%$ greater rate of cardiovascular events, after adjusting for depressive symptoms, age, disease severity, comorbid conditions, C-reactive protein, smoking, and medication adherence.

These findings raised the hypothesis that the increased risk of cardiovascular events associated with depression could potentially be preventable with behaviour modification. Especially exercise targets many of the mechanisms linking depression with the increased risk of cardiovascular events, including autonomic nervous system activity, hypothalamic-pituitary-adrenal (HPA) axis function, platelet activation, vascular function, and inflammation $[8,12]$.

\section{Depression as a Risk Factor for Type 2 Diabetes}

Evidence strongly suggests that depression is independent risk factor for the onset of type 2 diabetes. Knoll, Twisk, Beekman, Heine, Snoek, \& Pouwer examined the causal association among depression and type 2 diabetes by conducting a meta-analysis of longitudinal studies [13]. The pooled relative risk was 1.37; depressed adults have a $37 \%$ increased risk of developing type 2 diabetes. The most recent meta-analysis of Cosgrove, Sargeant, Griffin confirmed the causal role of depression or depressive symptoms in developing type 2 diabetes [14]. The pooled adjusted relative risk estimate from the 3 highest quality studies was 1.25. Twenty five percent of cases of diabetes could be attributed to depression in people with both conditions. Several pathophysiological mechanisms could explain the increased risk of type 2 diabetes in depressed individuals, including the increased activity of the HPA axis and sympathetic nervous system, and dysregulation of the immune system. Furthermore, physical inactivity, common in depressed individuals, is a strong risk factor for type 2 diabetes, independently of obesity [15]. On the other hand, physical activity improves glycemic control in type 2 diabetes, with greater improvements for combined aerobic and resistance training compared with aerobic or resistance training alone [16].

\section{Depression as a Risk Factor for Osteoporosis}

There is emerging evidence that depression is a risk factor for osteoporosis in both women and men [17,18]. A prospective study compared mineral bone density in 89 premenopausal women with depression and 44 healthy control women [17]. Low bone mass density was more prevalent in premenopausal women with depression. The bone mass density deficits were of clinical significance and comparable in magnitude to those resulting from established risk factors for osteoporosis, such as smoking and reduced calcium intake. The potential mechanism by which osteoporosis develops in depressed individuals are multifactorial. Endocrine and immune alternations secondary to both depression and osteoporosis play a pathogenic role in bone metabolism. In addition, unhealthy lifestyle factors associated with depression, including increased cigarette smoking and alcohol consumption, poor dietary patterns and physical inactivity have been shown to exert negative effects on bone mass density [19]. Regular exercise, especially resistance training, contributes to the development of bone mass. However, evi- dence suggests that the impact of exercise on osteoporosis is rather preventative than curative [20].

\section{EXERCISE IS MEDICINE}

There is clear scientific evidence proving the benefit of regular physical activity on both the primary and secondary prevention of type 2 diabetes, hypertension, heart disease, osteoporosis, and some cancers (particularly breast and colon cancer). Without a doubt, exercise really is medicine and it can be seen as the much needed vaccine to prevent chronic disease (inactivity-related diseases) and premature death [21]. On the other hand, physical inactivity is one of the most important public health problems of the 21 st century [22]. According to the World Health Organization, at least $60 \%$ of the global population fails to achieve the minimum recommendation for health-related physical fitness (i.e., for adults and older adults 30 minutes moderate to vigorous intensity activity daily, 5 days per week) [23].

\section{The Physical Health Problem of Physical Inactivity}

The Aerobics Center Longitudinal Study, a prospective epidemiological study, investigated health outcomes associated with physical activity and cardio-respiratory fitness at the Cooper Clinic, Dallas. The study estimated the attributable fraction of risk factors for death in a large population of 12.943 women and 40.842 men. The attributable fractions were adjusted for age and each other risk factor. The results showed that low cardiorespiratory fitness accounts for about $16 \%$ of all deaths in both women and men, and this was substantially more than that of obesity, diabetes, smoking and high cholesterol. Only hypertension in men was a higher risk factor [22].

The Aerobics Center Longitudinal Study also investigated the joint association of physical activity or fitness and obesity status with cardiovascular disease mortality in 2.316 men with type 2 diabetes. The average follow-up was 15.9 years. The results showed a strong inverse gradient for cardiovascular disease death across fitness categories within each BMI category. The researcher group emphasized that obese men who were moderately/highly fit had less than half the risk of dying than normal-weight men who were unfit [15].

\section{Physical (in)Activity and its Relation to Depression}

Goodwin investigated the relationship between lack of physical activity and depression using data from the National Co-morbidity Survey $(n=8098)$, a nationally representative sample of adults ages 15-54 in the United States [24]. Slightly over one-half of adults reported regular physical activity $(60.3 \%)$. Regular physical activity was associated with a significantly decreased prevalence of current major depression. Individuals who reported regular physical exercise were less likely to meet in the previous year criteria for diagnosis of major depression (8\% vs. 13\%). The association between regular physical activity and lower prevalence of current major depression $(\mathrm{OR}=0.75)$ persisted after adjusting for differences in socio-demographic characteristics, physical disorders, and co-morbid mental disorders. Physical activity also showed a dose-response relation with current major depression. 
Some prospective longitudinal studies suggest that physical activity is associated with a reduced risk of developing depression. In a sample of 4.848 subjects, Camacho, Roberts, Lazarus, Kaplan, Cohen reported that the lack of exercise was linked to later depression [25]. Paffenbarger, Lee, Leung found that physical activity negatively correlated with depression approximately 25 years later in a sample of 10.201 men [26]. In 2.084 elderly people, stratified into low and high depression, daily walking predicted improved depression in both the groups after 3 years [27]. The Aerobics Center Longitudinal Study investigated the association of cardio-respiratory fitness (CRF) with depressive symptoms in a cohort of 11.258 men and 3.085 women [28]. After an average of 12 years of follow-up, CRF fitness was inversely associated with the risk of developing elevated depressive symptoms. After adjustment for potential confounders, the relative risk of developing depressive symptoms were $31 \%$ lower for men with moderate CRF and $51 \%$ lower for men with high CRF compared to men with low CRF.

Regular physical exercise is significantly less common in women than in men and significantly less among those older than 44 [3]. The relationship between lack of regular physical activity and depression in middle-aged women has been the subject of two prospective cohort studies. The study of Brown, Ford, Burton, Marshall, Dobson examined the doseresponse relationship between physical activity and depressive symptoms in a cohort of 9.207 middle-aged women, corrected for pre-existing psychological and physical health problems [29]. The data suggested that there was a clear dose-response relationship between increasing physical activity and decreasing depressive symptoms, independent of pre-existing physical and psychological health. Lee and Russell reported on the longitudinal effects of physical activity and psychological well-being among Australian women [30]. Overall, women who had become physically active over a 3 year interval had positive changes in psychological wellbeing, while the opposite was true in those who had become less active.

Only one survey exclusively investigated a cohort of initially clinically depressed individuals $(n=424)$ [31]. Patients completed measures of depression, physical activity, exercise as coping strategy for depression, and other demographic and psychosocial constructs at baseline, and after 1year, 4-years, and 10-years follow-up. More physical activity was associated with less concurrent depression, even after controlling for gender, age, medical problems, and negative life events. In addition, physical activity counteracted the effects of medical conditions and negative life events on depression. The researchers concluded that physical activity may be especially helpful in the context of medical problems and major life stressors in depression.

\section{Exercise as Intervention for Depression}

There is growing evidence that exercise may be an effective therapy for mild to moderate depression and a valuable complementary therapy to the traditional treatments for severe depression. Meta-analytic studies provide one means of summarizing the primary research and identifying variables that may moderate the effects of exercise on depression. North, McCullagh, Tran analyzed 80 studies and reported an effect size of 0.53 (moderate effect) indicating that exercise training reduced depression scores by approximately onehalf a standard deviation as compared to the non-exercise groups; an even larger effect size (0.94) was reported in clinical populations [32]. Including only studies with patients diagnosed with major depression, Craft and Landers reported an effect size of 0.72 (moderate effect), showing that only the length of the exercise program was a significant moderator of the clinical effects, with programs of at least 9 weeks being associated with larger reductions in depression. Patient characteristics (age, gender, severity of depression) and type of exercise (aerobic versus resistance training) were not significant moderators. Exercise had comparable beneficial effects compared with standard treatment of depression (i.e. pharmacotherapy and psychotherapy) [33]. Limiting the analyses to randomized controlled trials $(n=14)$, Lawlor and Hopkins reported an effects size of 1.1 (large effect), when exercise training was compared to no-treatment control groups. In addition, exercise training was as effective as cognitive therapy [34]. The most recent meta-analysis of Cox included randomized controlled trials of exercise and follow-up with clinically depressed samples of older adults conducted between 2000-2006. Analyses looked at overall effect, duration, treatment studies and follow-up, outcome measures, and type of exercise. The overall effect size was moderate (0.46). Exercise was as effective as other standard treatment with reduction in levels of depression in all treatment conditions and little differences among conditions. Effect sizes were larger (0.52) during the treatment phase then during two years follow-up phase (0.30). No differences were found for type of exercise, aerobic, resistance or combined training [35].

A fundamental issue concerns the minimal effective dosage of exercise needed to improve depression. Dunn, Trivedi, Kampert, Clark, Chambliss studied the doseresponse relation of exercise and reduction in depressive symptoms in patients with mild to moderate major depression who exercised individually [36]. They found that individuals who exercised according to the public health recommendations, 3-5 times a week with a weekly energy expenditure of $17.5 \mathrm{kcal} / \mathrm{kg} /$ week, had significantly larger reductions in depression compared to those who exercised with low intensity and a weekly energy expenditure of 7 $\mathrm{kcal} / \mathrm{kg} / \mathrm{week}$. The latter regimen had results comparable to a placebo condition with stretching and flexibility exercise. After 12 weeks exercise according to the public health recommendations, the Hamilton Rating Scale for Depression (HRSD) scores were reduced $47 \%$ from baseline. Forty-six percent of participants in the public health recommendations group had a therapeutic response to treatment, defined as a $50 \%$ reduction in baseline HRSD, and $42 \%$ of this group had remission of symptoms, defined as an HRSD score $<7$. The researchers concluded that aerobic exercise, according to the public health recommendations, was effective in treating mild to moderate major depression. The rates of response and remission were comparable to the rates reported in trials of cognitive behavioural therapy, antidepressant medication, and other exercise studies.

Several physiological as well as psychological mechanisms mediate the effects of exercise on depression. The physiological include reduced activity of the HPA axis and decreased cortisol levels, and increased neurotransmission of 
monoamines and beta-endorphins [37-39]. The most pronounced psychological mechanisms are increased feelings of mastery or self-efficacy, distraction, a reduction in negative thought patterns, and social interaction.

\section{Exercise and Cognitive Decline}

Depression is associated with poor cognitive functioning. Being depressed is accompanied by slower information processing, psychomotor retardation, and poor memory functioning. There is growing evidence that exercise has a beneficial impact on cognitive function in older adults [39]. Positive effects of regular aerobic and resistance training on cognition have been observed in studies among subjects with and without cognitive decline [40,41]. The positive effects on cognition occur generally and even more so for those processes requiring greater executive control (i.e., processes involved in scheduling, planning, monitoring, and task coordination). Chronic exercise increases the expression of brain growth factors and may have neurogenerative and neuroprotective influences on the brain by stimulating the growth and development of new cells [42].

Kubesch, Bretschneider, Freudenmann, et al. studied the effect of single bouts of aerobic exercise on executive functions in depressed patients [43]. In a random order, 24 patients with major depressive disorder performed 30 minutes cycling at 2 different workload levels of $40 \%$ and $60 \%$ of their 4-mmol/L lactic acid exercise capacity. After the single 30-minute aerobic exercise bouts at both workload levels, positive effects on executive control processes were observed. The researchers suggested that the improvements were mediated via alteration of activity of the anterior cingulate cortex.

\section{CONCLUSION: EXERCISE PRODUCES SOME 'POSITIVE SIDE EFFECTS'}

A growing body of evidence suggests that exercise is an effective treatment for depression. For mild to moderate depression the effect of exercise may be comparable to antidepressant medication and psychotherapy; for severe depression exercise seems to be a valuable complementary therapy to the traditional treatments. Exercise training not only improves depression, but also produces 'positive side effects' on depression associated physical health problems and cognitive decline. Therefore, the effectiveness of exercise interventions for depression also needs to be evaluated in terms of preventing chronic disease and cognitive decline.

Psychiatrists, general practitioners and physical therapists are increasingly confronted with patients with depression. The World Health Organization expects that depression shows a rising trend during the coming 20 years. Research suggests a dose-response relationship between physical activity and both mental and physical health. On the other hand, physical inactivity is one of the most important public health problems of the 21 st century. At least $60 \%$ of the global population fails to achieve the minimum recommendation for health-related physical fitness. Due to the benefits of physical activity on depression and its co-morbid physical diseases and cognitive decline the promotion of physical exercise seems to be a crucial health promotion strategy. Physical therapists, with a specialised education in psychia- try, are ideally placed to motivate sedentary depressed individuals to become active and to stimulate physical activity as health promotion strategy.

Depressed patients accumulate a lot of barriers for participation in exercise programs such as: a low self-concept, loss of energy, interest and motivation, generalized fatigue, weak physical fitness and health condition, fear to move (movement phobia), social fear, being overweight, a low feeling of personal control concerning own fitness and health, and psychosomatic complaints. In order to improve patient's motivation and to optimize effects of exercise therapy following recommendations for physical therapists are helpful [44-45].

Anticipate the barriers for participation by an acquaintance conversation.

Give information about mental and physical health benefits of exercise. them.

Help the person find a form of physical activity that suits

Draw up an individual plan with the patient taking into account emotional, cognitive and physiological components of depression.

Create exercise programs based on initial physical fitness assessment and measurement of perceived exertion during exercise.

Formulate realistic objectives improving exercise compliance and motivation.

Adapt the moderate exercise stimulus to the individual's physical abilities, training status, expectations and goals, side effects of psychotropic medication, exercise tolerance and perceived exertion.

Follow the program with exercise cards and provide regular progress feedback to the patients.

Avoid between-patient comparisons.

Focus on perceived fitness gains, achievement of personal goals, mastery experiences and sense of control over the body and its functioning.

\section{REFERENCES}

[1] American Psychiatric Association: diagnostic and statistical manual of mental disorders. 4th ed. Washington, DC: American Psychiatric Association 1994.

[2] Blumenthal J. Depression and coronary heart disease: association and implications for treatment. Cleve Clin J Med 2008; 75 (Suppl) 2: $48-53$

[3] World Health Organization: Prevention of mental disorders: effective interventions and policy option: Summary report. Geneva: World Health Organization 2004.

[4] Lett H, Blumenthal J, Babyak M, Sherwood A, Strauman T, Robins C. Depression as a risk factor for coronary artery disease: evidence, mechanisms, and treatment. Psychosom Med 2004; 66: 305-15.

[5] Rugulies R. Depression as a predictor for coronary heart disease. A review and meta-analysis. Am J Prev Med 2002; 23: 51-61.

[6] Rozanski A, Blumenthal J, Davidson K, Saab P, Kubzansky L. The epidemiology, pathophysiology, and management of psychosocial risk factors in cardiac practice: the emerging field of behavioural cardiology. J Am Coll Cardiol 2005 45: 637-51.

[7] Frasure-Smith N, Lesperance F, Talajic M. Depression following myocardial infarction. Impact on 6-month survival. JAMA 1993 270: 1819-25. 
[8] Zoeller R. Physical activity: depression, anxiety, physical activity, and cardiovascular disease: what's the connection? Am J Lifestyle Med 2007 1: 175-80.

[9] Strine W, Mokdad A, Dube S. The association of depression and anxiety with obesity and unhealthy behaviors among communitydwelling US adults. Gen Hosp Psychiatry 2007; 30: 127-37.

[10] Kamphuis M, Geerlings M, Tijhuis M, et al. Physical inactivity, depression, and risk of cardiovascular mortality. Med Sci Sports Exerc 2007; 39: 1693-9.

[11] Whooley M, de Jonge P, Vittinghoff E, et al. Depressive symptoms, health behaviors, and risk of cardiovascular events in patients with coronary heart disease. JAMA 2008; 300: 2379-88.

[12] Martinsen E, Raglin J. Themed review: anxiety/depression. Am J Lifestyle Med 2007; 1: 159-66.

[13] Knol M, Twisk J, Beekman A, Heine R, Snoek F, Pouwer F. Depression as a risk factor for the onset of type 2 diabetes mellitus. A meta-analysis. Diabetologia 2006; 49: 837-45.

[14] Cosgrove M, Sargeant L, Griffin S. Does depression increase the risk of developing type 2 diabetes? Occup Med 2008: 58; 7-14.

[15] Church T, LaMonte M, Barlow C, Blair S. Cardiorespiratory fitness and body mass index as predictors of cardiovascular disease mortality among men with diabetes. Arch Intern Med 2005; 165: 2114-20.

[16] Sigal R, Kenny G, Boulé N, et al. Effects of aerobic training, resistance training, or both on glycemic control in type 2 diabetes. A randomized trial. Ann Intern Med 2007; 147: 357-69.

[17] Eskandari F, Martinez P, Torvik S, et al. Low bone mass in premenopausal women with depression. Arch Intern Med 2007; 167: 2329-36.

[18] Ilias I, Alesci S, Gold P, Chrousos G. Depression and osteoporosis in men: Association or casual link? Hormones 2006; 5: 9-16.

[19] Williams L, Pasco J, Jacka F, Henry M. Depression and bone metabolism, a review. Psychother Psychosom 2009; 78: 16-25.

[20] Siegrist M. Role of physical activity in the prevention of osteoporosis. Med Monatsschr Pharm 2008; 31: 259-64.

[21] Physical Activity Guidelines Advisory Committee. Physical activity guidelines advisory committee report 2008. Washington, DC: US Department of Health and Human Services 2008.

[22] Blair S. Physical inactivity: the biggest public health problem of the 21st century. Br J Sports Med 2009; 43: 1-2.

[23] World Health Report. Geneva: World Health Organization; 2002.

[24] Goodwin R. Association between physical activity and mental disorders among adults in the United States. Prev Med 2003; 36: 698-703.

[25] Camacho T, Roberts R, Lazarus N, Kaplan G, Cohen R. Physical activity and depression: evidence from the Alamada County study. Am J Epidiomiol 1991; 134: 220-31.

[26] Paffenbarger R, Lee I, Leung R. Physical activity and personal characteristics associated with depression and suicide in American college men. Acta Psychiatr Scand 1994; (Suppl 377): 16-22.

[27] Mobily K, Rubinstein L, Lemke J, O'Hara M, Wallace R. Walking and depression in a cohort of older adults: The Iowa 65+ Rural Health Study. J Aging Phys Act 1996; 4: 119-35.
[28] Sui X, Laditka J, Church T, et al. Prospective study of cardiorespiratory fitness and depressive symptoms in women and men. J Psychiatr Res 2009; 43: 546-52.

[29] Brown W, Ford J, Burton N, Marshall A, Dobson A. Prospective study of physical activity and depressive symptoms in middle-aged women. Am J Prev Med 2005; 29: 265-72.

[30] Lee C, Russell A. Prospective study of physical activity and depressive symptoms in middle-aged women. J Psychosom Res 2003; 54: $155-60$.

[31] Harris A, Cronkite R, Moos R. Physical activity, exercise coping, and depression in a 10-year cohort study of depressed patients. J Affect Disord 2006; 93: 79-85.

[32] North T, McCullagh P, Tran Z. Effect of exercise on depression. Exerc Sport Sci Rev 1990; 18: 379- 415.

[33] Craft L, Landers D. The effect of exercise on clinical depression and depression resulting from mental illness: A meta-analysis. J Sport Exerc Psychol 1998; 20: 339-57.

[34] Lawlor D, Hopker, S. The effectiveness of exercise as an intervention in the management of depression: Systematic review and metaregression analysis of randomised controlled trials. BMJ 2001; 322 763-7.

[35] Cox J. Is exercise an evidence-based intervention for clinical depression in older adults: a meta-analysis of randomized studies 2000-2006. Florida: Florida State University 2007.

[36] Dunn A, Trivedi M, Kampert J, Clark C, Chambliss H. Exercise treatment for depression: Efficacy and dose response. Am J Prev Med 2005; 28: $1-8$.

[37] Duman C, Schlesingera L, Terwilligera R, Russell D, Newtona S, Duman R. Peripheral insulin-like growth factor-I produces antidepressant-like behaviour and contributes to the effect of exercise. Behav Brain Res 2008; 198: 366-71.

[38] Dishman R, O'Connor P. Lessons in exercise neurobiology: The case of endorphins. Mental Health and Physical Activity (in press).

[39] Dishman R, Berthoud H, Booth F, et al. Neurobiology of exercise. Obesity 2006; $14: 345-56$.

[40] Erickson K, Kramer A. Aerobic exercise effects on cognitive and neural plasticity in older adults. Br J Sports Med 2009; 43: 22-4.

[41] Liu-Ambrose T, Donaldson M. Exercise and cognition in older adults: is there a role for resistance training programmes? $\mathrm{Br} \mathrm{J}$ Sports Med 2009; 43: 25-7.

[42] Cotman C, Berchtold N, Christie L. Exercise builds brain health: key roles of growth factor cascades and inflammation. Trends Neurosci 2007; 30: 464-72.

[43] Kubesch S, Bretschneider V, Freudenmann R, et al. Aerobic endurance exercise improves executive functions in depressed patients. J Clin Psychiatry 2003; 64: 1005-12.

[44] Knapen J, Van de Vliet P, Van Coppenolle H, et al. Comparison of changes in physical self-concept, global self-esteem, depression and anxiety following two different psychomotor therapy programs in non-psychotic psychiatric inpatients. Psychother Psychosom 2005; 74: 353-61.

[45] Knapen J, Van de Vliet P, Van Coppenolle H, et al. Evaluation of cardio-respiratory fitness and perceived exertion for patients with depressive and anxiety disorders: A study on reliability. Disabil Rehabil 2003; 25: 1312-5.

Received: March 17, 2009

Revised: May 20, 2009

Accepted: June 03, 2009

(C) Knapen et al.; Licensee Bentham Open.

This is an open access article licensed under the terms of the Creative Commons Attribution Non-Commercial License (http://creativecommons.org/licenses/by-nc/3.0/) which permits unrestricted, non-commercial use, distribution and reproduction in any medium, provided the work is properly cited. 